\title{
A proposed genetic algorithm to optimize service restoration in electrical networks with respect to the probability of transformers failure
}

\begin{abstract}
Power system reliability, stability and efficiency are the most important issues to insure continuously feeding of customers. However in process of time, system will be age and the probability of failures will increase and faults inevitably will occur. When a fault occurs, the first reaction is isolation of the faulty area, then with aid of software and/or skillful person quick restoration is essentially needed. To minimize the out-of-service area and activity time of restoration many methods are suggested depend on objectives and constraints of restoration strategy. In many researches a Genetic Algorithm is employed as a powerful tool to solve this multi-objective, multi-constraint optimization problem. Out-of-service area minimization, reduce the number of switching operation and minimizing the minimum electrical power loss in restored system are the prior objectives of restoration plan. In this paper, as transformers are the most expensive and more effective equipments in the electrical network, failure probability increasing is introduced as a new constraint in genetic algorithm by authors. Expected results of this new algorithm should lead to a new plan of restoration in permissible ranges of transformer loading in respect of their age, previous experienced faults and condition monitoring.
\end{abstract}

Keyword: Electrical power system; Transformer failure; Genetic algorithm; Restoration 